International Journal of Computing, Communications and Networking

Available Online at http://www.warse.org/ijccn/static/pdf/file/ijccn03922020.pdf

https://doi.org/10.30534/ijccn/2020/03922019

\title{
Optimizing Sensor Network in Sustainable City
}

\author{
Gargy Ponnachan ${ }^{1}$, Drisya Merin Saji ${ }^{2}$, Bismi Nazar $^{3}$, Jossy P Xavier ${ }^{4}$, Dr. Vinodh P Vijayan ${ }^{5}$ \\ ${ }^{1}$ Mangalam College of Engineering, Kottayam, India, gargyp98@gmail.com \\ ${ }^{2}$ Mangalam College of Engineering, Kottayam, India, drisyamerin25@mail.com \\ ${ }^{3}$ Mangalam College of Engineering, Kottayam, India, bisminazar98@ gmail.com \\ ${ }^{4}$ Mangalam College of Engineering, Kottayam, India, jossypxavier@gmail.com \\ ${ }^{5}$ Mangalam College of Engineering, Kottayam, India,vinodh.pvijayan@mangalam.in
}

\begin{abstract}
A well monitored sustainable city enhances the lifestyle of the citizens by meeting their own needs with minimal natural resource consumption. The wireless sensor network plays an important role in collecting, processing and analyzing the corresponding information. We propose a system to monitor a city using various IoT enabled wireless sensor networks. With the help of IoT, smart city monitoring can be made possible through real-time monitoring, ubiquitous sensing and intelligent information processing. For best services, to exchange and communicate information IoT embedded systems can be used. It defines a customized design of IoT based monitoring devices. The sensors we use in the system efficiently monitor the city and collect information. The whole system is designed such that it helps in enhancing the condition of a city in many aspects. These information are used to predict upcoming environmentalconditions.
\end{abstract}

Key words : Energy efficient, Green city, Hybrid optimization, IoT, PSO, Raspberry Pi, WSN.

\section{INTRODUCTION}

Sustainable cities will have a huge demand in future. So it is very important to monitor the city to keep it sustainable. In a sustainable city, a massive number of sensors and devices are interconnected using IoT to help dealing with issues like air pollution, waste management, forest fire and energy efficiency[1]. The high exploitation of natural resources and increasing pollution are disturbing the ecosystem as well as the normal living conditions. Monitoring and maintaining a city are more challenging tasks than creating or building them[3]. So, monitoring using an energy efficient IoT enabled wireless network and data analytics will improve the overall performance of the system[5]. The entire city is covered with various sensors according to the parameters to be monitored. The sensors can be temperature sensor, humidity sensor, etc. These sensors monitor the city periodically to collect information and store it in a database. The collected information will be of huge size so classification is needed to distinguish useful information. These data can be used for predicting upcoming environmental conditions of the city.

\section{LITERATURE REVIEW}

\section{(i). Weather MonitoringSystem:}

Some digital and analog sensors are used in this system to measure the environmental parameters. This data from the input sensors will then be read by the server, i.e. Raspberry $\mathrm{Pi}$ and stored in CSV as well as text files. The sensors collect data of various environmental parameters and provide it to Raspberry Pi which acts as a base station. The Raspberry Pi then transmits the data using WiFi and it will be displayed on the laptop[8].

\section{(ii). Building Monitoring System using RaspberryPi:}

The main objective of this paper is to utilize Raspberry Pi as the main ingredient of the Building Monitoring System to monitor the environmental parameters and also to monitor the power consumption of the building environment inorder to develop an effective environment monitoring system which can be used correctly to execute data analytics for future energy harvesting[5].

\section{(iii). Methodology for Monitoring Manufacturing} Environment by using WSN andIoT:

This paper has developed for designing a WSN with the aim of monitoring manufacturing environments. Also, this method is a more seamless integration of WSN and the IoT. A case study of temperature monitoring in an office environment was presented for demonstrating and proving the proposedmethodology[6].

\section{(iv). IoT based Real Time Environment MonitoringSystem:}

This paper describes a design operative prototype based on IoT concepts for real time monitoring of various environmental conditions using certain commonly available and low cost sensors. The various environmental conditions are continuously monitored, processed and controlled by an Arduino Uno microcontroller board with the help of several sensors. Captured data arebroadcasted 
through the internet with the help of an ESP8266 WiFi module. The projected system delivers sensor data to an API called ThingSpeak over an HTTP protocol and allows storing of data. The prototype has been used to monitor and analyse real time data using graphical information of the environment[10].

\section{(v). WSN Using Raspberry Pi and Arduino for} Environment MonitoringApplications:

This paper describes a wireless sensor network system with Arduino, Raspberry Pi and a number of open-source software packages. The system has a number of features including low cost, easy to deploy and easy to maintain. Some sample deployment and measurement results are presented to demonstrate the usefulness of the system[9].

\section{SYSTEM ARCHITECTURE}

The objective of the project is to create an efficient monitoring system that monitors a sustainable city. The efficient monitoring can ensure earlier prediction of environmental hazards[4]. The city is covered with a variety of sensors based on the parameters to be monitored. These sensors collect data in a regular interval of time. The city contains a variety of places, these places are categorized based on its sensitivity towards environmental pollution, sound pollution, air pollution, water pollution, temperature, humidity, etc[5,6,7]. The sensors collect data and store it in a database. The collected data is of huge size and we can classify data to separate the useful information from the huge collected data. The output can be displayed on a laptop/desktop. This extracted data is analyzed and reached in a final conclusion. The collected data can be considered for detailed data analytics in order to identify the overall scenario in the ecosystem, help us to predict the future performance, which helps the authority to plan the necessary precautions and thereby ensure the best performance of the sustainable city[8].

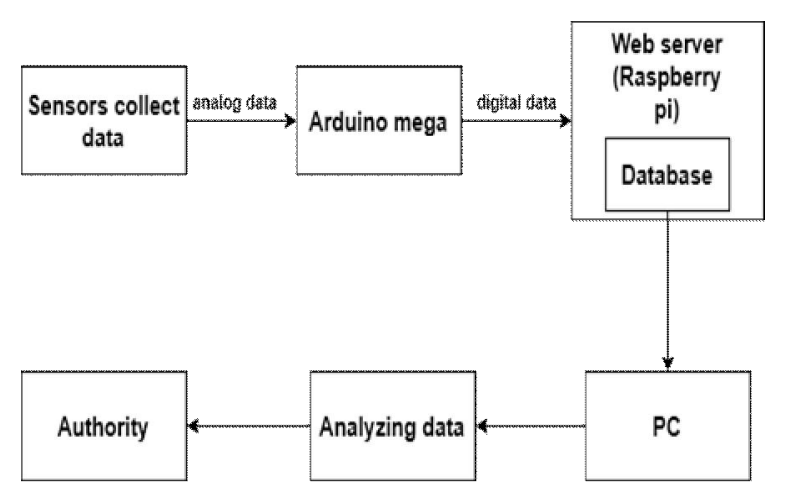

Figure 1: System architecture

\section{MODULES}

There are 5 modules:

- Sensors

- Arduinomega

- Web Server(Raspberrypi)

- PC

- Dataanalysis

\section{A. Sensors}

Here we use 5 sensors. These sensors are used for collecting data.

\section{(i) RainSensor}

A rain sensor or rain switch is a switching device activated by rainfall. There are two main applications for rain sensors. The first is a water conservation device connected to an automatic irrigation system that causes the system to shut down in the event of rainfall. The second is a device used to protect the interior of an automobile from rain and to support the automatic mode of windscreen wiper.

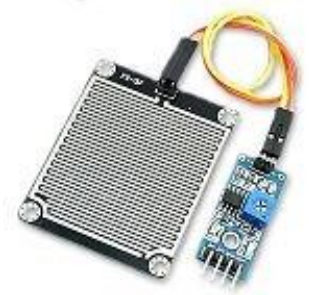

Figure 2: rain sensor

\section{(ii) SoundSensor}

It is used to detect the intensity of sound. This sensor employs a microphone to provide input to the buffer, peals detector and an amplifier[4]. This sensor notices a sound and processes an output voltage signal to a microcontroller. After that, it executes required processing.

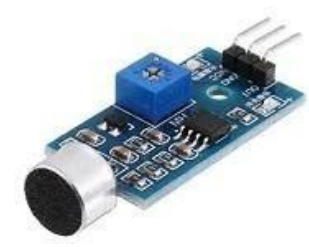

Figure 3: sound sensor 


\section{(iii) SmokeDetector}

It is a device that senses smoke, typically used as an indicator of fire.

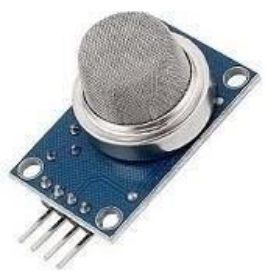

Figure 4: smoke detector

\section{(iv) BMP180}

It is designed to measure barometric pressure or atmospheric pressure. It is a high precision sensor designed for consumer applications. Barometric pressure is nothing but the weight of air applied on everything. The air has weight and wherever there is air its pressure is felt. BMP 180 sensor senses that pressure and provides that information in digital output.

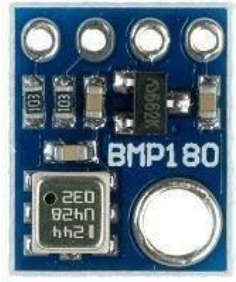

Figure 5: BMP 180

\section{(v) DHT11}

It is used as a temperature and humidity sensor. The sensor comes with a dedicated NTC to measure temperature and an 8 bit microcontroller to output the values of temperature and humidity as serial data. The sensor is also factory celebrated and hence easy to interface with other microcontrollers.

\section{B. ArduinoMega}

The data collected by sensors are of analog form which cannot be directly given to the Raspberry $\mathrm{Pi}$, so to convert the analog data to digital data we use an arduino[8].Instead of using separate arduino for each sensor we are using a single arduino called arduino mega. Arduino board is an open-source microcontroller board which is based on Atmega 2560 microcontroller[7]. The board includes digital input/output pins - 54, where 16 pins are analog input, 14 are used like PWM outputs, hardware serialports - 4, a crystal oscillator - $16 \mathrm{MHz}$, an ICSP header, a power jack, a USB connection as well as an RST button. This board mainly includes everything which is essential for supporting the microcontroller. So, the power suply of this board can be done by connecting it $\mathrm{t}$ o PC using a USB cable or battery or an AC - DC adapter. This board can be protected from the unexpected electrical discharge by placing a base plate.

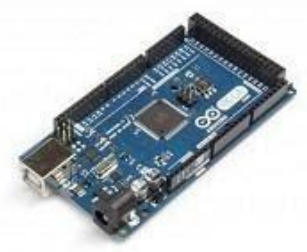

Figure 7: Arduino mega

\section{RaspberryPi}

The raspberry pi is an inexpensive and miniature computer. Here it acts as a web server. The data collected by sensors are stored in the Pi database. The input to $\mathrm{Pi}$ is digital, so we need to convert the analog data from sensors to digital using an analog to digital convertor(arduino mega). The raspberry pi board comprises a program memory(RAM), processor and graphics chip, CPU, GPU, ethernet port, GPIO pins, Xbee socket, UART, power source connector and various interfaces for other external devices[7]. It also requires mass storage for that we use an SD flash memory card. It is inserted into the slot on the board and acts as the hard drive for the Pi. The output can be viewed on a PC.

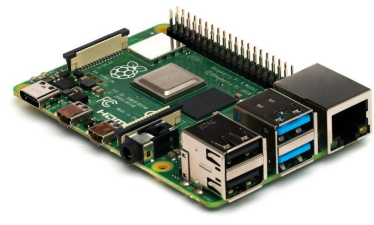

Figure 8: raspberry pi 
D. $P C$

The data collected from sensors are stored in the Pi database and the output can be viewed on a PC. The collected data is of huge size and we can filter them based on our requirement.

\section{E. DataAnalysis}

It is the scientific process of discovering and communicating the meaningful patterns which can be found in data. The data collected from the sensors are examined and any problem found is reported to the corresponding authority[5]. For eg, if we receive more rain on the data we collect, we need to notify the concerned authority about the possibility of flooding. The authority will take necessaryactions.

\section{EXPERIMENTAL ANALYSIS}

The sensor nodes collect environmental data at preset time and upload it to the Pi cloud. The data in the cloud can be displayed on the web interface. With the aim to check the reliability of the design, certain experiments are performed and results derived. The following graph shows the data collected by smoke and sound sensors. The data in the graphs are obtained by extracting the maximum concentration values from all the values collected from different sensors. The $x$ axis represents the time and $y$ axis represents concentration.

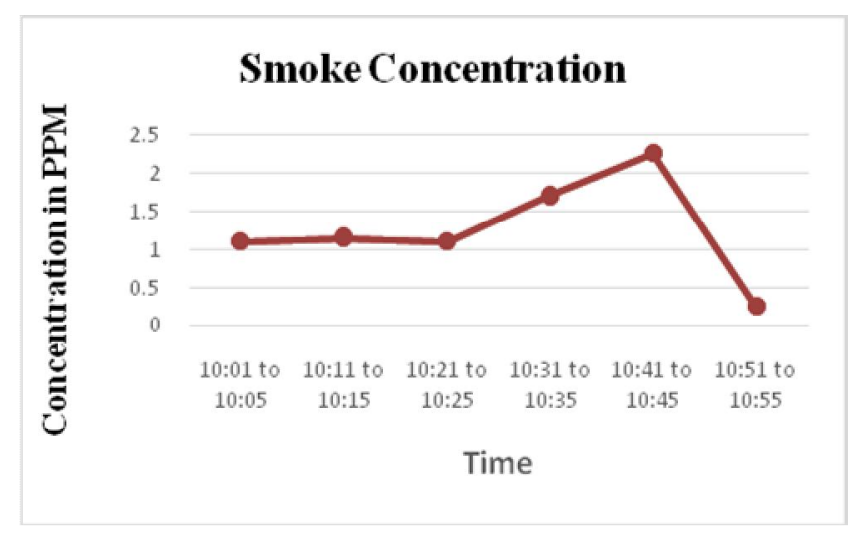

Figure 9: A sample data of smoke concentration

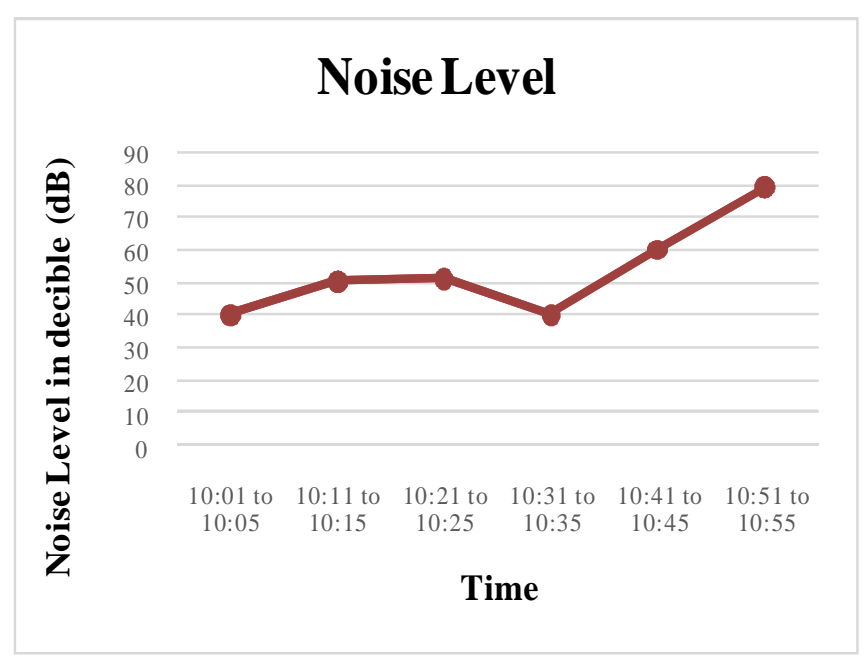

Figure 10: A sample data of noise level

Now these data have been stored in the cloud. The sensor data can be downloaded from the web interface. The data can be used for post processing and analysis of the parameter and continuous monitoring purpose.

\section{CONCLUSION}

In this project, we introduce an efficient IoT enabled wireless sensor network to monitor sustainable cities. A city is a place where people with different interests, requirements and expectations live. Monitoring a city is an important and challenging task which keeps the city sustainable. Our system monitors the city using variousIoT enabled sensors and stores it in a database. The collected data can be used for predicting the future performance of the city. It also helps us to predict the disasters and help us to take necessary actions and they ensure the best performance of the city. The temperature, pressure levels can be clearly identified based on this system at the device location. The internet enabled device is capable of monitoring the quality of air, rain, temperature, pressure, etc. Further modifications can be done to this by using some scheduling algorithms to schedule the monitoring. Continuous monitoring may decrease the battery life of the system.

\section{REFERENCES}

[1] Weidang Lu, Yi Gong, Xin Liu, Jiayingwu, "Collaborative Energy and Information Transfer in Green Wireless Sensor Networks for Smart Cities", IEEE Transactions on Industrial Informatics, DOI 10.1109/TII.2017.2777846. 
[2] Li Ping Qian, Yuan Wu, Bo Ji, Liang Huang, Danny H.K.Tsang, "Hybrid IoT: Integration of Hierarchical Multiple Access and Computation Offloading for IoTBased Smart Cities", IEEE Transactions on Industrial Informatics,0890-8044/19/2019.

[3] Zhou Su, Qinghua Zheng, Bo Dong, Qichao Xu, Minnan Luo, "Secure Content Delivery with Edge Nodes to Save Caching Resources for Mobile users in Green Cities", IEEE Transactions on Industrial Informatics, DOI 10.1109/TII.2017.2787201.

[4] Vinodh P Vijayan, Deepti John, Merina Thomas, Neetha V Maliackal, Sara Sangeetha Varghese "Multi Agent Path Planning Approach to Dynamic Free Flight Environment", International Journal of Recent Trends in Engineering (IJRTE), ISSN 1797-9617 Volume 1, Number 1, May 2009, Page(s): 41-46.

[5] Juby Joseph, Vinodh P Vijayan" Misdirection Attack in WSN Due to Selfish Nodes; Detection and Suppression using Longer Path Protocol" International Journal of Advanced Research in Computer Science and Software Engineering, Volume 4, Issue 7, July 2014, pp. 825-829, ISSN: 2277 128X

[6] V P Vijayan, Biju Paul "Multi Objective Traffic Prediction Using Type-2 Fuzzy Logic and Ambient Intelligence" International Conference on Advances in Computer Engineering 2010, Published in IEEE Computer Society Proceedings, ISBN: 978-0-7695-4058-0, Print ISBN: 978-1-4244-7154-6

[7]Vijayan V P, Gopinathan E "Improving Network Coverage and Life-Time in a Cooperative Wireless mobile Sensor Network " Fourth International Conference on Advances in computing and communications (ICACC) Aug, 2014. Published in IEEE Computer Society Proceedings. Print ISBN: 978-1-47994364-7,

INSPEC

AccessionNumber:14630874,DOI:10.1109/ICACC.2014.1 6 PP 42-45.

[8] Vinodh P Vijayan, Biju Paul “ Traffic scheduling for Green city through energy efficient Wireless sensor Networks" International Journal of Advanced Trends in Computer Science and Engineering, Volume 8, No.4, July - August 2019, ISSN 2278-3091, https://doi.org/10.30534/ijatcse/2019/81842019. 